\title{
DIAGNOSTIC ABILITY OF BARRETT'S INDEX TO DETECT DYSTHYROID OPTIC NEUROPATHY USING MULTIDETECTOR COMPUTED TOMOGRAPHY
}

\author{
Mário L. R. Monteiro, Allan C. P. Gonçalves, Carla T. M. Silva, Janete P. \\ Moura, Carolina S. Ribeiro, Eloisa M.M.S. Gebrim
}

doi: $10.1590 / \mathbf{S 1 8 0 7 - 5 9 3 2 2 0 0 8 0 0 0 3 0 0 0 0 3}$

\begin{abstract}
Monteiro MLR, Gonçalves ACP, Silva CTM, Moura JP, Ribeiro CS, Gebrim EMMS. Diagnostic ability of Barrett's index to detect dysthyroid optic neuropathy using multidetector computed tomography. Clinics. 2008;63:301-6.
\end{abstract}

OBJECTIVES: The objective of this study was to evaluate the ability of a muscular index (Barrett's Index), calculated with multidetector computed tomography, to detect dysthyroid optic neuropathy in patients with Graves' orbitopathy.

METHODS: Thirty-six patients with Graves' orbitopathy were prospectively studied and submitted to neuro-ophthalmic evaluation and multidetector computed tomography scans of the orbits. Orbits were divided into two groups: those with and without dysthyroid optic neuropathy. Barrett's index was calculated as the percentage of the orbit occupied by muscles. Sensitivity and specificity were determined for several index values.

RESULTS: Sixty-four orbits (19 with and 45 without dysthyroid optic neuropathy) met the inclusion criteria for the study. The mean Barrett's index values $( \pm$ SD) were $64.47 \% \pm 6.06 \%$ and $49.44 \% \pm 10.94 \%$ in the groups with and without dysthyroid optic neuropathy, respectively $(p<0.001)$. Barrett's index sensitivity ranged from $32 \%$ to $100 \%$, and Barrett's index specificity ranged from $24 \%$ to $100 \%$. The best combination of sensitivity and specificity was $79 \% / 72 \%$ for BI=60\% (odds ratio: 9.2 ).

CONCLUSIONS: Barrett's Index is a useful indicator of dysthyroid optic neuropathy and may contribute to early diagnosis and treatment. Patients with a Barrett's index $\geq 60 \%$ should be carefully examined and followed for the development of dysthyroid optic neuropathy.

KEYWORDS: Dysthyroid Optic Neuropathy. Multidetector computed tomography. Graves' Orbitopathy. Visual loss.Thyroid eye disease.

\section{INTRODUCTION}

Optic nerve dysfunction is a debilitating complication of Graves' orbitopathy (GO) occurring in $5-8.6 \%$ of patients, ${ }^{1}$ and it is almost always a result of compression of the optic nerve at the orbital apex caused by enlarged extraocular muscles. Symptoms and signs of dysthyroid optic neuropathy (DON) may include decreased visual acuity (VA), abnormal

Division of Ophthalmology and the Departments of Endocrinology and Radiology, Hospital das Clínicas da Faculdade de Medicina da Universidade de São Paulo - São Paulo, Brazil.

mlrmonteiro@terra.com.br

Received for publication on October 10, 2007

Accepted for publication on January 15, 2008 visual fields (VF), altered color and brightness perception, afferent pupillary defects, and edema or atrophy of the optic nerve head. ${ }^{1}$ When the majority of these symptoms and signs are present, the diagnosis of DON is straightforward. It is, however, much more difficult to establish when changes in VA are subtle since such changes may be related to a number of other factors, including decreased corneal transparency due to exposure keratopathy. ${ }^{2}$ Furthermore, many tests for optic nerve dysfunction, such as VA, color and brightness perception and VF, especially when performed with standard automated perimetry (SAP), require full cooperation of an alert and motivated patient. Also, such tests will not infrequently render false positive results, particularly in patients with congestive GO, leading to diagnostic confusion. Objective tests capable 
of rapidly and accurately identifying patients prone to DON development are therefore highly desirable.

Computerized tomography (CT) studies in patients with DON often reveal enlargement of extraocular muscles at the orbital apex, and several authors have correlated the degree of apical crowding on CT scans with optic neuropathy in an attempt to raise suspicion of DON and facilitate its diagnosis. ${ }^{3-5}$ In 1988, Barrett et al. ${ }^{5}$ reported findings for a muscular index for $\mathrm{CT}$ expected to reflect extraocular muscle impingement of the optic nerve in patients with GO. The authors calculated the percentage of orbital width occupied by muscles (muscular index) from coronal and axial cuts halfway between the posterior aspect of the globe and the apex of the orbit. A muscular index of $67 \%$ or greater was reported to be indicative of compressive optic neuropathy. ${ }^{5}$ Nevertheless, the difficulties in obtaining perfectly oriented coronal cuts and in defining the exact position of the coronal cut along the length of the orbit make the technique inappropriate for routine use.

The introduction of multidetector computed tomography (MDCT) has resulted in significantly increased scanning speed and reduction of artifacts and errors in the evaluation of measurements of orbital contents, as the images may be reformatted in any plane with high resolution. ${ }^{6}$ Calculating structure indices and volumes is relatively simple and may be performed at a workstation connected to the equipment. Thus, the purpose of this prospective study was to evaluate the value of Barrett's index (BI) used in combination with MDCT as a predictor of DON in patients with GO.

\section{MATERIAL AND METHODS}

Between July 2005 and June 2007, 36 patients with GO were prospectively studied. GO was diagnosed in accordance with previously reported criteria. ${ }^{7}$ The study followed the principles of the Declaration of Helsinki, and approval was granted by the Institutional Review Board Ethics Committee. All patients underwent a complete neuro-ophthalmic examination, including best-corrected VA, applanation tonometry, pupillary reactions, extraocular motility evaluation, slit lamp examination, evaluation of eyelid and soft tissue inflammation, measurement of the lid fissure, Hertel exophthalmometry, fundoscopy, and VF evaluation.

VF was performed using manual perimetry and standard automated perimetry (SAP). Manual VF testing was performed using the Goldmann perimeter (GP) (HaagStreit AG, Bern, Switzerland). The V-4-e, I-4-e, I-3-e, I-2-e and I-1-e stimuli were used to draw the isopters. Kinetic determinations were followed by static presentation of the stimuli particularly in the central 30-degree area, in order to search for localized defects. SAP was performed with a Humphrey field analyzer 750 (Zeiss-Humphrey, Dublin, CA) using a $4-\mathrm{mm}^{2}$ Goldmann size III stimulus $\left(0.43^{\circ}\right)$ on a dim background (31.5 apostilb). The central field was measured by a threshold 24-2 test program that presents stimuli on a $6^{\circ}$-spaced grid encompassing the central $21^{\circ}$ of the VF (24-2 SITA Standard test). The patients' appropriate near correction was used. Rest breaks were allowed when requested. To qualify as an abnormal VF on SAP, three adjacent abnormal points at $\mathrm{P}<0.05$ or two adjacent points with one abnormal point at $\mathrm{P}<0.01$ were required. ${ }^{8}$

Both orbits of each patient were scanned on a 16-slice MDCT scanner (Brilliance 16; Philips Medical Systems, Nederland B.V., the Netherlands) without the use of sedation or intravenous contrast. Scanning was performed with the patients' eyes closed and immobile. The MDCT scan parameters were: $\mathrm{Kv}=120 ; \mathrm{mAs}=200$; detector configuration $=16 \times 0.75 \mathrm{~mm}$; slice thickness $=1.5 \mathrm{~mm}$; image spacing $=0.7 \mathrm{~mm}$. We used the method described by Barrett et al. ${ }^{5}$ to generate a muscular index capable of quantifying extraocular muscle impingement on the optic nerve. A reformatted coronal scan halfway between the posterior globe and the orbital apex was used to calculate the muscular index. The transverse dimensions of the medial rectus muscle (A), the lateral rectus muscle (B), and the orbital width $(\mathrm{C})$ were measured along a horizontal line through the optic nerve. The horizontal muscular index was calculated as the percentage of orbital width occupied by the lateral and medial rectus muscles $[(\mathrm{A}+\mathrm{B} / \mathrm{C}) .100]$ (Figure 1). The vertical muscle index was based on the vertical dimensions of the superior rectus/levator muscle complex (D), the inferior rectus muscle (E), and the orbital height $(\mathrm{F})$ [(D+E/F).100] (Figure 1). The larger value was considered the muscular index, corresponding to the Barrett Index (BI), and was used in subsequent data analysis ${ }^{5}$. Measurements were made by an examiner that was not aware of the presence or absence of optic neuropathy.

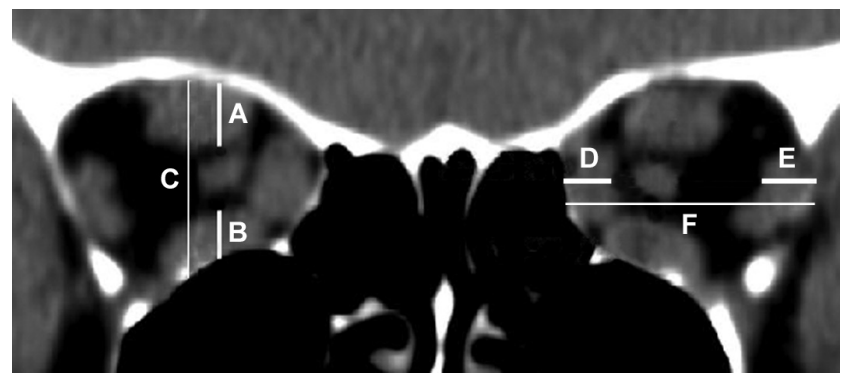

Figure 1 - Schematic representation of the method for calculating Barrett's Index. The vertical index is calculated by the sum of the vertical muscle diameters (A and $B$ ) divided by the vertical dimension of the orbit $(\mathrm{C})$. The horizontal index is calculated by dividing the sum of the horizontal muscle diameters (D and E) by the horizontal diameter of the orbit $(\mathrm{F})$. 
Sixty-four of the 72 orbits scanned were selected for the study. Orbits of eyes with GO and visual function data clearly indicating the presence or absence of active DON were eligible. The diagnostic criteria for DON were the presence of a relative afferent pupillary defect and the presence of a confirmed VF defect on SAP or GP associated or not with diminished best-corrected VA not caused by changes in transparency. When VA was normal and VF abnormalities were present on SAP, a repeat examination using SAP or GP was performed to rule out false positive responses. Thus, only eyes with confirmed VF defects were included in the analysis. Eyes with normal VA and nonreproducible VF abnormalities upon repeat examinations were excluded. Patients with ocular or optic nerve diseases that prevented the correct diagnosis of DON were also excluded. All patients with DON had an active disease, but less than four weeks of onset of visual disturbance and clinical signs of orbital congestive disease.

The sensitivity and specificity of BI for identifying eyes with DON was calculated in the conventional manner. A criterion with a high specificity, if positive, "rules in" the disease, and a test with a high sensitivity, if negative, "rules out" the disease. ${ }^{9}$ After carefully determining the presence or absence of DON, we calculated the sensitivity and specificity (with $95 \%$ confidence interval) for a number of pre-defined criteria for BI index values, including 40\%, 50\%, 60\%, 67\%, and $70 \%$. The odds ratio for having DON was calculated for each of these values.

We used an unpaired t-test for comparisons between groups of orbits with and without DON and Fisher's exact test for evaluating the significance of sensitivity and specificity values. Adherence to normal distribution was assessed using the Shapiro-Wilk test. $P$ values of less than 0.05 were considered significant.

\section{RESULTS}

Sixty-four orbits from 36 patients with GO were included in the analysis. Nineteen of these were from 12 patients ( 7 women and $5 \mathrm{men}$; mean age \pm SD: $53.3 \pm 10.4$ years) who met the diagnostic criteria for DON. Seven patients had bilateral DON, and both orbits were included in the study. Five patients had only one orbit included in the group of DON; in two patients, the contralateral eye did not meet the criteria for DON; two patients had the contralateral eye excluded, since the presence or absence of DON could not be clearly determined; one patient had a history of central retinal artery occlusion unrelated to $\mathrm{GO}$, which led to our exclusion of the contralateral eye. Forty-five orbits were affected with GO but not with DON; two of these were from patients with DON in the contralateral eye, while 43 were from 24 patients (17 women and 7 men; mean age \pm SD: $45.5 \pm 15.2$ years) with DON absent in both eyes. Five orbits in this group were excluded from the study: in three, the diagnosis of GO could not be established (unilateral GO); in one the eye was deviated in an extreme upward position, and therefore it was not possible to determine either the presence or absence of DON; and in one an old orbital fracture was observed.

Of the 19 orbits of eyes with DON, VA was 1.0 in $11,0.9$ in $4,0.8$ in 3 and 0.6 in one. The mean $( \pm \mathrm{SD})$ of SAP mean deviation was $-7.48 \pm 5.26 \mathrm{~dB}$. Results from the glaucoma hemifield test were outside the normal range in 16 eyes and borderline in three. Exophthalmometry measurements in affected eyes ranged from 19 to $30 \mathrm{~mm}$ (mean \pm SD: 24.66 $\pm 3.08 \mathrm{~mm}$ ). Restrictive myopathy was present in all orbits with DON. Fundoscopic examination revealed optic disc edema in a single eye and no abnormalities in the remainder of the eyes. In the group without DON, VA was 1.0 in all eyes and exophthalmometry findings ranged from 20.0 to $28.0 \mathrm{~mm}$ (mean \pm SD: $23.2 \pm 2.1$ ).

The mean $\mathrm{BI} \pm \mathrm{SD}$ for the groups with and without DON was $62.67 \% \pm 8.14 \%$ and $49.44 \% \pm 10.94 \%$, respectively $(p<0.001)$. Table 1 shows the relative frequency of orbits calculated with BI values of $40 \%, 50 \%, 60 \%, 67 \%$, and $70 \%$ in the 64 orbits studied. Table 2 summarizes the sensitivity and specificity for the respective BI values. The sensitivity ranged from $32 \%$ to $100 \%$, and the specificity ranged from $24 \%$ to $100 \%$. The odds ratio was statistically significant for all values of BI values tested (Table 2). The best combination of sensitivity/specificity was $79 \% / 72 \%$ for $\mathrm{BI}=60 \%$, yielding an odds ratio of 9.2 .

Table 1 - Relative frequency of orbits according to Barrett's Index, in 64 orbits with Graves' Orbitopathy (GO) with and without dysthyroid optic neuropathy (DON)

\begin{tabular}{lcc}
\hline Barrett Index & $\begin{array}{c}\text { GO with DON } \\
(\mathrm{n}=19)\end{array}$ & $\begin{array}{c}\text { GO without DON } \\
(\mathrm{n}=45)\end{array}$ \\
\hline $40 \%$ & $19(100.0 \%)$ & $34(75.6 \%)$ \\
$50 \%$ & $19(100.0 \%)$ & $23(51.1 \%)$ \\
$60 \%$ & $15(78.9 \%)$ & $13(28.9 \%)$ \\
$67 \%$ & $8(38.1 \%)$ & $2(4.4 \%)$ \\
$70 \%$ & $6(28.6 \%)$ & $0(0 \%)$ \\
\hline
\end{tabular}

\section{DISCUSSION}

DON requires prompt diagnosis and treatment in order to prevent permanent damage to the visual function. Although DON can most often be diagnosed on clinical grounds based on visual function assessment, the presence of an afferent 
Table 2 - The sensitivity, specificity and odds ratios with confidence intervals (CI) of several levels of Barrett's index for the diagnosis of 19 orbits with dysthyroid optic neuropathy and of 45 orbits of patients with Graves' Orbitopathy without dysthyroid optic neuropathy

\begin{tabular}{lcccc}
\hline Barrett's Index & Sensitivity (95\% CI) & Specificity (95\% CI) & Odds Ratio & $\mathrm{P}^{*}$ \\
\hline $40 \%$ & $1.0(0.87-1.00)$ & $0.24(0.19-0.24)$ & $\operatorname{Inf}(1.51-$ Inf) & 0.025 \\
$50 \%$ & $1.0(0.86-1.00)$ & $0.49(0.43-0.49)$ & $\operatorname{Inf}(4.49-$-Inf $)$ & $<0.001$ \\
$60 \%$ & $0.79(0.61-0.91)$ & $0.72(0.64-0.76)$ & $9.2(2.66-31.57)$ & $<0.001$ \\
$67 \%$ & $0.42(0.27-0,49)$ & $0.96(0.89-0.99)$ & $15.6(3.19-73.97)$ & 0.001 \\
$70 \%$ & $0.32(0.20-0.32)$ & $1.0(0.95-1.00)$ & Inf $(4.95-$-Inf $)$ & $<0.001$ \\
\hline
\end{tabular}

* Fisher's exact test. Significant values are in italics. Inf $=$ infinity.

pupillary defect and fundoscopic abnormalities such as disc edema and choroidal folds, the diagnosis of DON can be difficult in many cases. The uncertainty in the diagnosis occurs mainly in non-cooperative patients or those who have difficulties in performing psychophysical tests such as SAP, which is particularly common in anxious patients with marked proptosis, lid retraction, and profuse tearing. Therefore, additional objective data such as imaging findings can be very helpful in the diagnosis.

Although inflammatory and vascular mechanisms have also been suggested, the most widely accepted mechanism of optic neuropathy suggests that pathologically enlarged extraocular muscles compress the optic nerve at the orbital apex. In fact, several authors have documented the relationship between DON and the size of the extraocular muscles as measured by $\mathrm{CT}^{3,}, 5,10-14$ Other imaging features reflecting an increased volume of orbital contents, such as proptosis and apical crowding, have also been shown to be more common in patients with optic neuropathy. ${ }^{1,4}{ }^{14}$ Birchall et al..$^{15}$ suggested that $\mathrm{CT}$ evidence of fat herniating through the superior ophthalmic fissure would be a useful indicator of DON. However, in recent reports by McKeag et al., ${ }^{16}$ they observed this condition in only 40 of 94 eyes with DON, suggesting that it would have a low sensitivity as an indicator of DON. A gradation of apical crowding has been described by Nugent et al. ${ }^{4}$ These authors found apical crowding greater than $50 \%$ to be a useful indicator of DON, with a sensitivity of $66 \%$ and a specificity of $87 \%$. McKeag et al. ${ }^{16}$ confirmed the usefulness of this indicator in a report of apical crowding greater than $50 \%$ in 78 of 98 eyes with DON. However, it should noted that measuring apical crowding or the muscle occupation tax at the orbital apex is often a cumbersome task, since the muscle limits can be difficult to identify. Extraocular muscles, the superior orbital vein, and the optic nerve are frequently indistinct at the orbital apex even when high resolution CT is used.

Barrett et al. ${ }^{5}$ described what is probably the easiest and most reproducible method for correlating the degree of extraocular muscle enlargement with the development of DON: a muscular index calculated as the percentage of orbital width occupied by vertical and horizontal muscles in relation to orbital dimensions as measured from the midpoint between the globe and the orbital apex. In a retrospective study, the authors concluded that a muscular index of $67 \%$, as encountered in 12 of 49 orbits evaluated, would be virtually diagnostic of DON. Recently, in another retrospective study evaluating an index of $67 \%$, Giaconi et al. ${ }^{14}$ found that $55 \%$ of orbits with DON and six out of 34 orbits without DON met the criterion. All 20 orbits affected with DON presented a muscular index greater than 50\%. In another retrospective study, Birchall et al. ${ }^{15}$ reported a sensitivity of $50 \%$ and a specificity of $73 \%$ for BI of $50 \%$. These authors observed that only one of the 17 orbits with DON had BI of $67 \%$, a result very different from those observed by Barrett et al., ${ }^{5}$ probably as a result of the inclusion of milder cases of DON.

To our knowledge, this is the first prospective study using MDCT to investigate the sensitivity and specificity of BI. Since MDCT can achieve very accurate reformations in the coronal plane, with a clear definition of the position along the orbit, we believe that the study shows a significant improvement in quality and reproducibility, compared to previous reports. Furthermore, in our study patients were carefully and prospectively evaluated for the presence or absence of DON. Our results indicate that if a percentage of $67 \%$ is required for diagnosis, as suggested by Barrett et al., 5 DON would be identified in only 8 out of 19 affected orbits. Moreover, two orbits without DON met the criterion, despite careful evaluation with SAP indicating absence of VF abnormalities. Thus, a muscular index of $67 \%$ presented a sensitivity of only $42 \%$ and a specificity of $96 \%$. In our study, when lower values of BI were tested, we observed a significant increase in sensitivity with a corresponding decrease in specificity. For example, when BI was $40 \%$, the sensitivity was $100 \%$ and the specificity, $24 \%$; with $\mathrm{BI}=$ $50 \%$, the sensitivity was $100 \%$ and the specificity, $49 \%$; with $\mathrm{BI}=$ $60 \%$, the sensitivity was $79 \%$ and the specificity, $72 \%$. Finally, for higher BI values, such as 70\%, we have found a sensitivity of $32 \%$ and a specificity of $100 \%$. The best combination for sensitivity/ specificity was therefore observed with $\mathrm{BI}=60 \%$. With this index, the odds ratio for the presence of DON was 9.2 (95\% confidence interval; 2.66 - 31.57).

Our study indicates a BI of $67 \%$ is neither required for nor an 
absolute indicator of the presence of DON. However, it is important to consider that BI could also vary depending on the severity of the neuropathy. For example, Guy et al. ${ }^{17}$ considered DON to be severe when visual acuity was equal to or worse than 20/80. Because we included patients with milder forms of DON, it is understandable that some of the subjects presented lower BI values. Furthermore, because $\mathrm{BI}$ is measured on the orbit halfway between the globe and the optic canal, it is likely that diagnoses in some patients with predominantly apical muscle enlargement are missed. Finally, it is important to point out that, although extraocular muscle enlargement is the most likely mechanism for the development of visual loss in DON, unusual cases of DON without extraocular muscle enlargement have been reported. ${ }^{18}$
Despite these limitations, we believe BI can be helpful when screening for DON because it is easily calculated for patients with GO submitted to MDCT. Our findings indicate that patients with a muscular index greater than $60 \%$ should be carefully examined for the presence of DON. Although high index values may be found in some patients with $\mathrm{GO}$ and normal visual function, the great majority of cases will have values associated with DON, and thus this assessment may contribute to early diagnosis and treatment.

\section{ACKNOWLEDGEMENTS}

Fundação de Amparo a Pesquisa do Estado de São Paulo, FAPESP (No 06.61337-9, Carla T. M. Silva), São Paulo, Brazil.

\section{REFERENCES}

1. Neigel JM, Rootman J, Belkin RI, Nugent RA, Drance SM, Beattie CW et al. Dysthyroid optic neuropathy. The crowded orbital apex syndrome. Ophthalmology. 1988;95:1515-21.

2. Kazim M, Trokel S, Moore S. Treatment of acute Graves orbitopathy. Ophthalmology. 1991;98:1443-8.

3. Kennerdell JS, Rosenbaum AE, El-Hoshy MH. Apical optic nerve compression of dysthyroid optic neuropathy on computed tomography. Arch Ophthalmol. 1981;99:807-9.

4. Nugent RA, Belkin RI, Neigel JM, Rootman J, Robertson WD, Spinelli $\mathrm{J}$, et al. Graves orbitopathy: correlation of CT and clinical findings. Radiology. 1990;177:675-82.

5. Barrett L, Glatt HJ, Burde RM, Gado MH. Optic nerve dysfunction in thyroid eye disease: CT. Radiology. 1988;167:503-7.

6. Chapman VM, Grottkau BE, Albright M, Salamipour H, Jaramillo D. Multidetector computed tomography of pediatric lateral condylar fractures. J Comput Assist Tomogr. 2005;29:842-6.

7. Bartley GB, Gorman CA. Diagnostic criteria for Graves' ophthalmopathy. Am J Ophthalmol. 1995;119:792-5.
8. Wall M, Neahring RK, Woodward KR. Sensitivity and specificity of frequency doubling perimetry in neuro-ophthalmic disorders: a comparison with conventional automated perimetry. Invest Ophthalmol Vis Sci. 2002;43:1277-83.

9. Sackett D, Haynes R, Guyatt G, Tugwell P. Clinical Epidemiology, 2 nd ed. Boston:Little, Brown \& Company. 1991:1-441.

10. Feldon SE, Weiner JM. Clinical significance of extraocular muscle volumes in Graves' ophthalmopathy: a quantitative computed tomography study. Arch Ophthalmol. 1982;100:1266-9.

11. Feldon SE, Muramatsu S, Weiner JM. Clinical classification of Graves' ophthalmopathy. Identification of risk factors for optic neuropathy. Arch Ophthalmol. 1984;102:1469-72.

12. Feldon SE, Lee CP, Muramatsu SK, Weiner JM. Quantitative computed tomography of Graves' ophthalmopathy. Extraocular muscle and orbital fat in development of optic neuropathy. Arch Ophthalmol. 1985;103:213-5.

13. Hallin ES, Feldon SE. Graves' ophthalmopathy: I. Simple CT estimates of extraocular muscle volume. Br J Ophthalmol. 1988;72:674-7. 
14. Giaconi JA, Kazim M, Rho T, Pfaff C. CT scan evidence of dysthyroid optic neuropathy. Ophthal Plast Reconstr Surg. 2002;18:177-82.

15. Birchall D, Goodall KL, Noble JL, Jackson A. Graves ophthalmopathy: intracranial fat prolapse on CT images as an indicator of optic nerve compression. Radiology. 1996;200:123-7.

16. McKeag D, Lane C, Lazarus JH, Baldeschi L, Boboridis K, Dickinson AJ, et al. Clinical features of dysthyroid optic neuropathy: a European Group on Graves' Orbitopathy (EUGOGO) survey. Br J Ophthalmol. 2007;91:455-8
17. Guy JR, Fagien S, Donovan JP, Rubin ML. Methylprednisolone pulse therapy in severe dysthyroid optic neuropathy. Ophthalmology. 1989;96:1048-52; discussion 52-3.

18. Anderson RL, Tweeten JP, Patrinely JR, Garland PE, Thiese SM . Dysthyroid optic neuropathy without extraocular muscle involvement. Ophthalmic Surg. 1989;20:568-74. 